\title{
Pipe flow with large particles and their impact on the transition to turbulence
}

\author{
Martin Leskovec $\odot,{ }^{1,{ }^{*}}$ Fredrik Lundell $\odot,{ }^{1,2}$ and Fredrik Innings $\oplus^{3,4}$ \\ ${ }^{1}$ FLOW, Department of Engineering Mechanics, KTH Royal Institute of Technology, \\ Stockholm SE-100 44, Sweden \\ ${ }^{2}$ Wallenberg Wood Science Center, Stockholm SE-100 44, Sweden \\ ${ }^{3}$ Department of Food Technology, Engineering and Nutrition, Lund University, Lund SE-221 00, Sweden \\ ${ }^{4}$ Tetra Pak Processing System AB, Lund SE-223 55, Sweden
}

(Received 3 April 2020; accepted 25 September 2020;

published 16 November 2020)

\begin{abstract}
The classical transition from laminar to turbulent flow is affected if solid particles are added. The transition behavior is a function of particle size $d$ and solid volume fraction $\phi$ and the flow undergoes a smooth transition, as opposed to intermittent, if $\phi$ exceeds a certain threshold. In this work we show that, for particle-laden pipe flows with large particle-to-pipe diameter ratios $d / D$, the $\phi$ threshold for altering the transition is much lower than previously reported for smaller particles. Magnetic resonance velocimetry reveals that particles introduce turbulent-like fluid velocity fluctuations in laminar flow. Factors that might control the limits between "classical" and "smooth" transition in the state space spanned by $d / D$ and $\phi$ are discussed based on scaling analyses.
\end{abstract}

DOI: 10.1103/PhysRevFluids.5.112301

Transition from laminar to turbulent flow in pipes has been investigated since the work of Reynolds [1] in 1883, who found three flow states with distinct characteristics: ordered flow, laminar, disordered flow, turbulent, and an intermediate transitional flow. In spite of nearly 140 years of intense investigations, transition has still not revealed all its secrets. For pipe flow, the flow state is governed by a sole parameter, the Reynolds number, which for a Newtonian fluid with density $\rho_{f}$ and dynamic viscosity $\mu$ in a pipe of diameter $D$ at mean bulk velocity $U_{b}$ is given as $\operatorname{Re}=\rho_{f} U_{b} D / \mu$. The transition process starts with the formation of turbulent "flashes" at a critical Reynolds number $\operatorname{Re}_{c}$. Reynolds [1] observed that $\operatorname{Re}_{c}$ varied in the range $2000 \lesssim \operatorname{Re}_{c} \lesssim 13000$ depending on inlet conditions. At present it is established that turbulence cannot be triggered below $\operatorname{Re} \approx 1000$ [2] and that laminar flow can be maintained up to $\operatorname{Re} \approx 10^{5}$ [3].

There are two types of turbulent flashes in the transitional regime: puffs and slugs [4-6]. If the Reynolds number is large enough, the puffs grow into faster traveling slugs that spread and lead to fully developed turbulence. This process is rather sudden and the change from a laminar to a turbulent state is distinct [7-9].

One way to determine the flow state in pipe flow is from the friction factor $f$. It is a dimensionless measure of the fluid friction, or drag, and is defined as $f=D \Delta P /\left(2 \rho U^{2} \Delta L\right)$ where $\Delta P / \Delta L$ is the pressure drop per unit length of pipe needed to overcome wall friction. The friction factor for laminar

\footnotetext{
*marles@kth.se
}

Published by the American Physical Society under the terms of the Creative Commons Attribution 4.0 International license. Further distribution of this work must maintain attribution to the author(s) and the published article's title, journal citation, and DOI. Funded by Bibsam. 
flow is $f_{\text {lam }}=16 /$ Re and for turbulent flow $f$ is satisfactorily estimated by empirical relationships such as the Blasius law $f_{\text {turb }}=0.0791 \mathrm{Re}^{-1 / 4}[10]$. The classical transition behavior in a Newtonian single-phase fluid is an intermittent mix of laminar and turbulent states with $f_{\text {lam }}$ as a lower bound and $f_{\text {turb }}$ as an upper bound. The average friction factor is a decreasing function of Re that follows $16 / \operatorname{Re}$ until $\operatorname{Re}_{c}$ when it rather abruptly increases and shows a local maximum at the end of the transition region, whereafter it decreases as $f \propto \mathrm{Re}^{-1 / 4}$ [9].

Matas et al. [11] showed that the addition of particles to a Newtonian carrier fluid alters the transition behavior and that $\operatorname{Re}_{c}$ is a nonmonotonic function of particle volume fraction $\phi$ and particle size $d$. Particles smaller than $d / D \approx 1 / 65 \approx 0.015$ delay transition and larger particles promote transition to turbulence compared with single-phase flow. Numerical studies by Yu et al. [12] of the same flow cases replicated the findings. Here, the authors pointed out that it is difficult to judge whether the flow is laminar or turbulent since particles induce local disturbances, which lead to nonsmooth flow even in the laminar regime and there is no longer a sudden increase in fluctuations. Recent experiments by Hogendoorn and Poelma [13] and Agrawal et al. [14] indicate that, for particles of similar size as studied by Matas et al. [11], the classical behavior of laminar-turbulent transitions with a sudden and abrupt increase in $f$ occurs only at $\phi \lesssim 17 \%$. At higher $\phi$, the transition behavior of the friction factor is smooth: $f$ has no local maximum in the transition region and turbulent puffs are difficult to distinguish from the globally fluctuating flow.

Apart from altering the transition behavior of the friction factor, the addition of particles also modulates the turbulence. Several length scales can be defined in turbulent flow, two being (i) the smallest length scale, the Kolmogorov length scale $\eta=\left(v^{3} / \varepsilon\right)^{1 / 4}$, estimated from kinematic viscosity $v$ and energy dissipation per unit mass $\varepsilon$ and (ii) the integral length scale $l_{e}$, which is the characteristic length of the most energetic eddies. Particles smaller than $\eta$ will have little effect on the turbulence production and dissipation. For $\eta<d \lesssim 0.1 l_{e}$ turbulence is attenuated and, for $d \gtrsim 0.1 l_{e}$, turbulence is augmented. The main mechanism for attenuation is increased dissipation from particle drag, and augmentation is driven by velocity fluctuations arising from particle wakes $[15,16]$.

Another effect of particles is additional stresses compared with the case of single-phase flow: in a turbulent flow of a pure fluid, viscous and Reynolds stresses exist, but, for a particle-laden flow, a third type of stress, particle-stress, is added. Lashgari et al. [17] simulated a particle-laden channel flow and found three different flow regimes: laminar-like at low Re and low $\phi$ where viscous stresses dominate; turbulent-like at high Re where Reynolds stresses dominate; and inertial shearthickening at high $\phi$ where particle-stresses dominate. In 1954 Bagnold [18] showed that, as $\phi$ increases, the stress from turbulent fluctuations in the fluid decreases and will eventually give way for particle stress. To determine the state of the suspension, he introduced the Bagnold number $\mathrm{Ba}=4 \operatorname{Re}_{p, \dot{\gamma}} \lambda^{1 / 2}$, where $\operatorname{Re}_{p, \dot{\gamma}}=\rho_{f} \dot{\gamma} d^{2} / \mu$ is the particle Reynolds number based on shear rate at the wall $\dot{\gamma}$ and $\lambda=1 /\left[(0.74 / \phi)^{1 / 3}-1\right]$ is the linear concentration which represents the particle diameter to particle spacing ratio; $\mathrm{Ba}$ is the ratio of inertial to viscous stresses.

In this work we experimentally investigate the transition behavior of suspensions with large particles $(d / D \geqslant 0.17)$ and show that the friction factor $f$ has no local maximum and transition is gradual instead of abrupt, even at $\phi=5 \%$. We perform a scaling analysis of agitation by particles and viscous dissipation and propose a curve separating "classical" and "smooth" transitions in the $\phi$ vs $d / D$ state space.

The experimental setup consists of a circulating loop with a straight and horizontal cylindrical pipe, a holding tank where the suspension is introduced, a centrifugal pump, and a magnetic resonance imaging scanner. The inner diameter of the pipe is $D=33.5 \pm 0.15 \mathrm{~mm}$ and the measurement section is $6 \mathrm{~m}$ long, corresponding to $180 D$. To calculate the friction factor $f$, the pressure drop is measured by using a differential pressure transducer (FKC11, 0-6 kPa, Fuji Electric, France) with an accuracy of $0.1 \%$ of the span. Pressure is measured at two points separated by a distance $L=40 D$, where the first pressure tap is located $100 \mathrm{D}$ downstream of a $180^{\circ}$ pipe bend. The second pressure tap is located $140 \mathrm{D}$ downstream the bend. The length of the pipe and the location of the pressure taps are sufficient to ensure fully developed laminar flow. The mean volumetric flow rate is measured by 
TABLE I. Three sets of particles. Material is PMMA (procured commercially) and Nylon PA12 (3Dprinted).

\begin{tabular}{lrcccc}
\hline \hline Case & \multicolumn{1}{c}{$d(\mathrm{~mm})$} & $d / D$ & $\phi_{\max }$ & $\rho_{p}\left(\mathrm{~kg} / \mathrm{m}^{3}\right)$ & Material \\
\hline$S S$ & $5.70 \pm 0.07$ & 0.17 & 0.58 & 1188 & PMMA \\
$L S$ & $13.30 \pm 0.09$ & 0.40 & 0.52 & 1156 & PMMA \\
$C$ & $6.95 \pm 0.08$ & 0.20 & 0.60 & 1038 & Nylon PA12 \\
\hline \hline
\end{tabular}

using an electromagnetic flow meter (Krohne Optiflux 1000, IFC 300, Krohne Messtechnik GmbH, Germany). To reduce the risk of measurement errors, the flow rate was also measured by collecting a sample of the suspension under a given time. This was done for the extreme case of high particle content and high $\mathrm{Re}$, and the error is within $2.5 \%$. The mean flow velocity $U$ is calculated from the mean flow rate. The flow is driven by a centrifugal pump (Flygt model 3085.183, Xylem Water Solutions AB, Sweden) with a modified impeller and volute in order to allow passage of particles. To ensure the accuracy of the experimental rig, $f$ was measured for single-phase flow and it matched well with $16 / \mathrm{Re}$ in the laminar regime and $0.0791 \mathrm{Re}^{-1 / 4}$ in the turbulent regime for a range of flow rates and fluid viscosities.

Three sets of particles (see Table I) were used in the experiments, two spherical and one cubical. The small spherical particles $(S S)$ were $5.70 \mathrm{~mm}$ and the large $(L S) 13.30 \mathrm{~mm}$ in diameter. The cubical, dice-shaped particles $(C)$ were $6.95 \mathrm{~mm}$ along the sides.

Different carrier fluids were prepared (see Table II) where density-matching with the particles was achieved by adding sugar (10-45 wt.\%) and the viscosity was further modified by adding carboxymethylated cellulose (CMC) (0.5-1.5 wt.\%). The viscosity of the carrier fluid was measured before experiments by using a rheometer (TA AR-2000ex, TA Instruments, Inc., USA) and during experiments using a viscometer (DV-II+ Pro, AMETEK Brookfield, USA). Due to its high sugar content, the carrier fluid viscosity was sensitive to temperature changes, which is why the temperature of the solution was maintained at a constant level by an external heat exchanger in the holding tank. To account for added particles, an effective viscosity $\mu_{e}$ was used for determining the effective Reynolds number $\operatorname{Re}_{e}$. To compute $\mu_{e}$, Eiler's viscosity model [19] was used: $\mu / \mu_{e}=\left(1+1.25 \frac{\phi}{1-\phi / \phi_{\max }}\right)^{2}$.

We used a $1 \mathrm{~T}$ magnetic resonance system (Aspect Imaging, Israel) to obtain carrier fluid mean velocity and estimate variance of the fluid velocity through magnetic resonance velocimetry (MRV). The details of the measurement technique and the MR system can be found in Dyverfeldt et al. [20], Elkins and Alley [21], and MacKenzie et al. [22].

The main results are presented in Figs. 1 and 2. In Fig. 1(a) the friction factor as a function of $\mathrm{Re}_{e}$ for all cases in Table III show a similar behavior: collapse onto a line slightly above the single-phase $f=16 / \operatorname{Re}_{e}$ for $\operatorname{Re}_{e}<800$ and a collapse onto the turbulent Blasius line for $\operatorname{Re}_{e}>5000$. In between, all particle-laden cases demonstrate a monotonic connection between the two lines. Compared with single-phase flow (blue crosses) the increase in $f$ at constant Re based

TABLE II. Fluids used and experimental range of Reynolds number.

\begin{tabular}{lccccc}
\hline \hline Case & ${ }^{\circ}$ Brix & CMC $($ wt. $\%)$ & $\rho\left(\mathrm{kg} / \mathrm{m}^{3}\right)$ & $\mu(\mathrm{mPa})$ & $\mathrm{Re}$ \\
\hline$S S_{1}$ & 43.3 & 0.5 & 1190 & 40 & $50-1750$ \\
$S S_{2}$ & 36.2 & 0 & 1155 & 4.4 & $3000-20000$ \\
$L S_{1}$ & 36.8 & 0.5 & 1157 & 38 & $50-2000$ \\
$L S_{2}$ & 36.0 & 0 & 1150 & 4.2 & $3000-20000$ \\
$C_{1}$ & 10.4 & 1.5 & 1040 & 35 & $50-2000$ \\
$C_{2}$ & 49.8 & 0 & 1225 & 14 & $1500-6000$ \\
\hline \hline
\end{tabular}



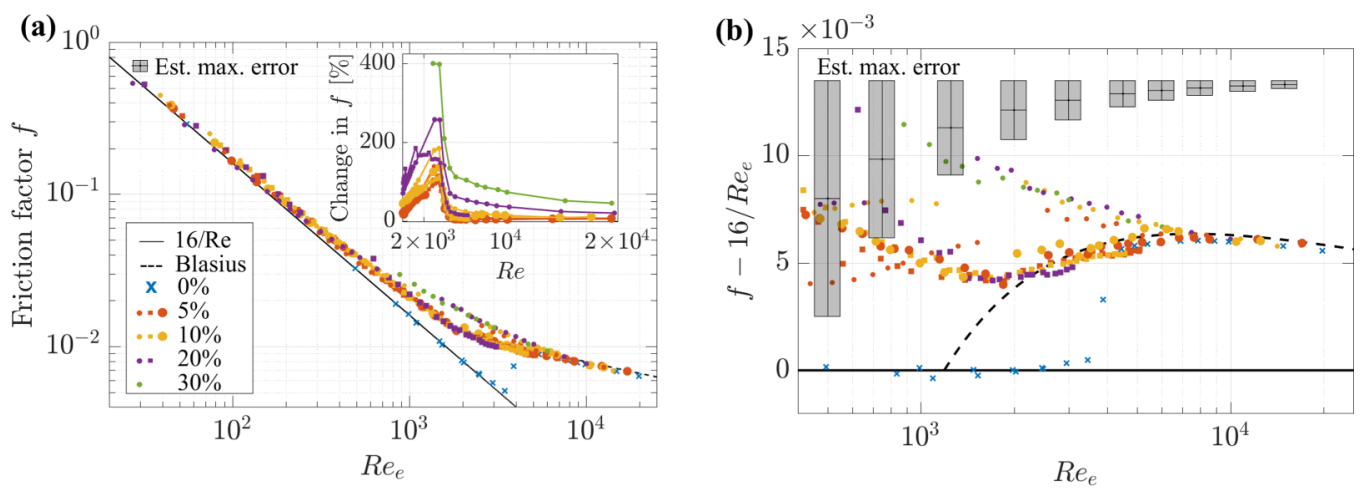

FIG. 1. (a) Friction factor $f$ as a function of $\operatorname{Re}_{e}$ for all cases and pure water, as indicated in the legend. The inset shows relative increase in $f$ at constant Reynolds number based on carrier fluid viscosity (constant $\mathrm{Re})$. The increase in the inset is the increase experienced by adding particles to a fluid at constant total flow rate. (b) The data in panel (a) is plotted as $f-16 / \operatorname{Re}_{e}$ to highlight deviations from laminar single-phase flow. Conservative estimates of the maximum errors are illustrated as gray regions [in panel (b), the error depends on $\mathrm{Re}_{e}$ ]. Symbols and lines are the same in panels (a) and (b).

on fluid viscosity can be up to $400 \%$ when particles are added, see inset of Fig. 1(a). The transition from a laminar to a turbulent state for $400<\mathrm{Re}_{e}<20000$ is scrutinised in more detail in Fig. 1(b), where $f-16 / \operatorname{Re}_{e}$ is plotted. Also, it is clear here that the particle-laden cases have an increased friction factor compared with single-phase flow at low $\mathrm{Re}_{e}$ and approach the fully turbulent curve from above. This is in contrast with the single-phase data, which approaches the turbulent Blasius curve by a distinct transition from below. All particle-laden cases demonstrate a smooth transition, and the friction factor approaches the turbulent curve from above. Interestingly, there seems to be two paths along which the friction factor approaches the turbulent curve: an upper one for the small-sphere cases and a lower one for the large-sphere and cube cases. This observation might be the footprint of changes in the (most probably very rich) underlying dynamics, which have yet to be further scrutinised, described, and understood.

The estimated maximum errors are shown in Figs. 1(a) and 1(b) as gray highlighted regions. A conservative estimate gives maximum relative errors in $\mathrm{Re}_{e}$ and $f$ to be $9.5 \%$ and $8.5 \%$, respectively. In the log-log plot of Fig. 1(a) the relative error is the same regardless of $\operatorname{Re}_{e}$ and $f$. The maximum absolute error for $f-16 / \operatorname{Re}_{e}$ is $\operatorname{Re}_{e}$ dependent and decreases as $\operatorname{Re}_{e}$ increases, which is seen in Fig. 1(b). The maximum errors indicated in Figs. 1(a) and 1(b) are extremely conservative and is based on the worst case in all measured variables. The actual errors are probably considerably smaller, given the agreement between the single-phase data and the $f=16 / \operatorname{Re}_{e}$ line. For $\operatorname{Re}_{e}>$ 1000 , the two curves for the friction factor are separated also when the very conservative error estimate is accounted for.

The profiles for mean and variance of velocity are presented in Fig. 2. Mean velocity profiles at $\mathrm{Re}=700$ in Figs. 2(a), 2(d), and 2(g) change from laminar to turbulent-like as $\phi$ increases from $0 \%$ (blue) to $20 \%$ (purple). The corresponding variance profiles in Figs. 2(b), 2(e), and 2(h) show that significant fluctuations are present already at this subcritical Re for the particle-laden cases. For a given particle the variance profiles are similar for $350<\operatorname{Re}<2000$, as seen in Figs. 2(c), 2(f), and 2(i), with an increase in amplitude as Re is increased. Generally, the particles seem to induce fluctuations in a region reaching a particle diameter away from the wall at $r / R=1$.

When the flow is dominated by viscous stresses, at low $\operatorname{Re}$ and low $\phi$, fluctuations will be dampened out. As $\phi$ is increased, the fluctuations increase, stemming from particle wakes, and the flow is in a nonstationary state where the energy injected into the flow is balanced by increased fluid dissipation. Apparently, this gives rise to an increase in $f$ compared with single-phase flow and 
(a)

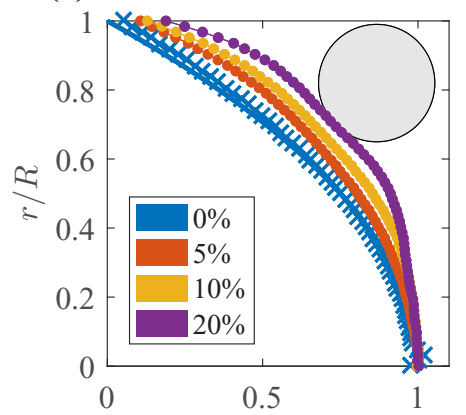

(d)

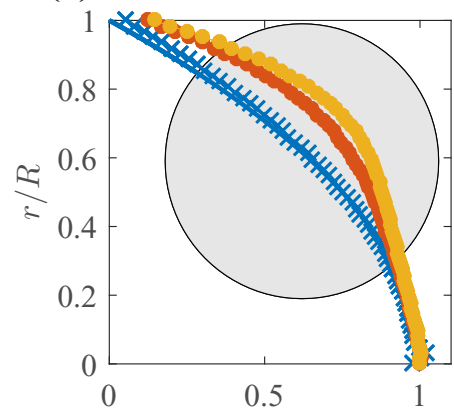

(g)

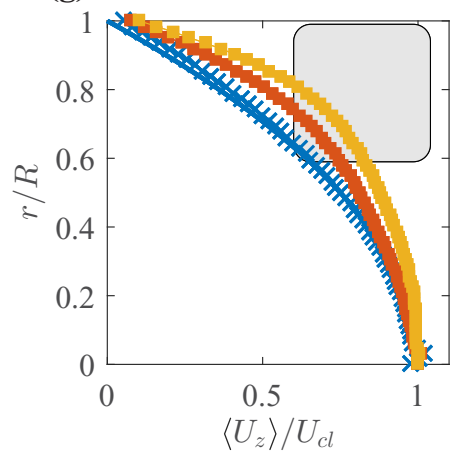

(b)

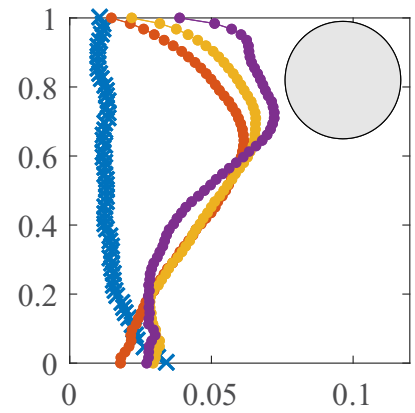

(e)

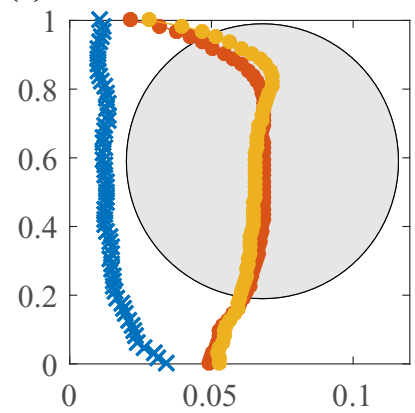

(h)

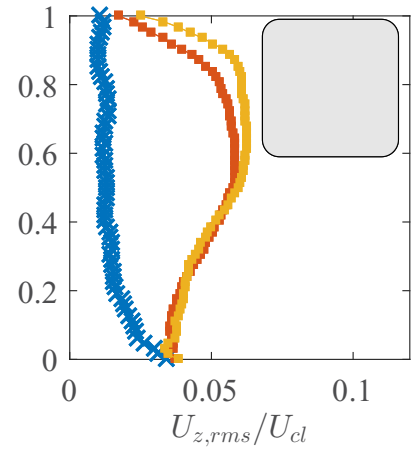

(c)

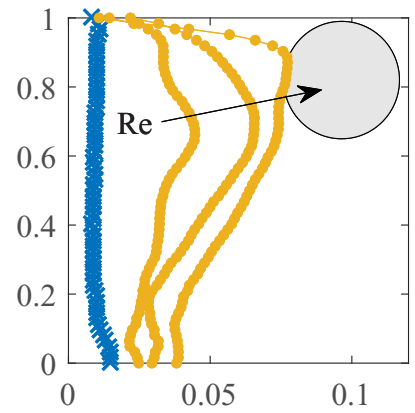

(f)

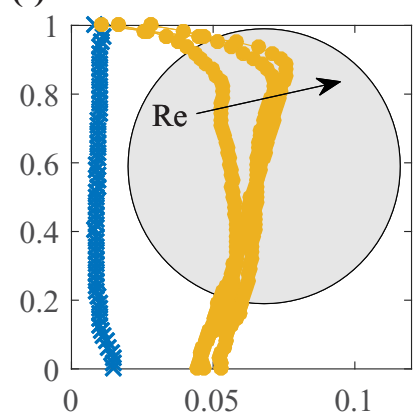

(i)

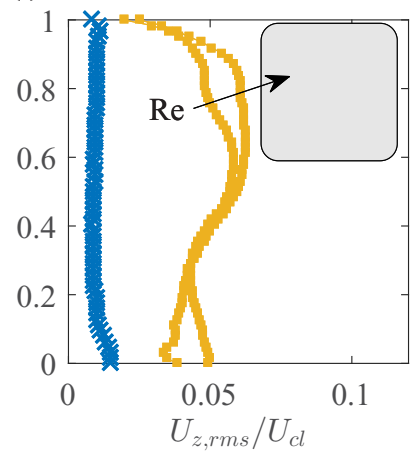

FIG. 2. (a), (d), (g) Mean axial velocity at $\mathrm{Re}=700$. (b), (e), (h) Variance of velocity at $\operatorname{Re}=700$. (c), (f) Variance of velocity at $\mathrm{Re}=350,700$, and 2000. (i) Variance of velocity at $\mathrm{Re}=350$ and 700 . Top row $S S$, middle row $L S$, and bottom row $C$. A flattening of the mean profiles at the center of the pipe can be seen with increasing volume concentration, indicating a turbulent-like flow. Peak in the variance indicate aggregation of particles at given $r / R$. The particle size in each case is indicated with gray schematics.

a smooth transition scenario, as seen in Fig. 1. To find the limits of particle size $d / D$ and volume concentration $\phi$ that will give a classical or smooth transition, we employ a scaling analysis coupled with experimental observations from our own work and the work of Hogendoorn and Poelma [13] and Agrawal et al. [14]. The results are plotted in Fig. 3.

To drive turbulent dynamics, the particle-induced fluctuations (or agitation) must be (i) strong enough and (ii) have a physical size that is large enough. We will now address (i) by comparing the amplitude of particle agitation to the fluid dissipation in particle-free flow. Neighboring particles translating under shear will be displaced in the radial direction when passing each other, and at a 
TABLE III. Range of Reynolds number for different particles and volume fractions.

\begin{tabular}{lcccc}
\hline \hline Case & $5 \%$ & $10 \%$ & $20 \%$ & $30 \%$ \\
\hline$S S$ & $50-20000$ & $50-20000$ & $50-20000$ & $3000-20000$ \\
$L S$ & $50-20000$ & $50-20000$ & & \\
$C$ & $50-6000$ & $50-6000$ & $50-6000$ & \\
\hline \hline
\end{tabular}

given concentration $\phi$, the frequency of particle-particle interactions scales as $\dot{\gamma} \phi$. A rough estimate of the work needed to displace a particle during such a passage is $F_{D} d$, where $F_{D}$ is the drag on a particle. This work can be obtained as $C_{D} \frac{1}{2} \rho(\dot{\gamma} d)^{2} \frac{\pi}{4} d^{3}$ by using the standard definition of the drag coefficient $C_{D}$ (assumed to be of order unity for this scaling analysis). Thus, the agitation power for each particle (work of a single-particle passage divided by time between passages) should scale as $\rho \dot{\gamma}^{3} d^{5} \phi$. This can be compared with the total dissipation in particle free laminar pipe flow, which scales as $\mu L U^{2}$. Since the number of particles in a pipe section scales as $\phi L D^{2} / d^{3}$, the fluid dissipation per particle scales as $\mu U^{2} d^{3} / D^{2} \phi$. From these estimates, the ratio between particle agitation and laminar dissipation is obtained as $C=\phi^{2}(d / D)^{2} \operatorname{Re}$, where $C$ is a dimensionless coefficient.

The blue solid line in Fig. 3 shows the curve that passes $d / D=1 / 19.5$ and $\phi=0.17$ for which this ratio is constant (at a constant Reynolds number). The red curve for constant Bagnold number (i.e., constant effect of particle collisions) and the blue line (constant relative agitation, introduced above) show similar trends. However, for $d / D>0.06$, the curves diverge and if the shift from classical transition to smooth is due to particle collisions, one should not see classical transitions at all for $d / D>0.15$. If, however, the shift is governed not by particle collisions but by particle agitation, classical transitions should be observed also for very large particles at sufficiently low concentrations. When it comes to (ii), the physical size of the particle-induced fluctuations, they can be compared with the dissipative length scale of the flow, $\eta=\left(v^{3} / \varepsilon\right)^{1 / 4}$. Since the dissipation in the fluid is given by the pumping power per unit volume, the equation $d / \eta=(d / D)(4 \sqrt{2} \operatorname{Re})^{-1 / 2}$ can

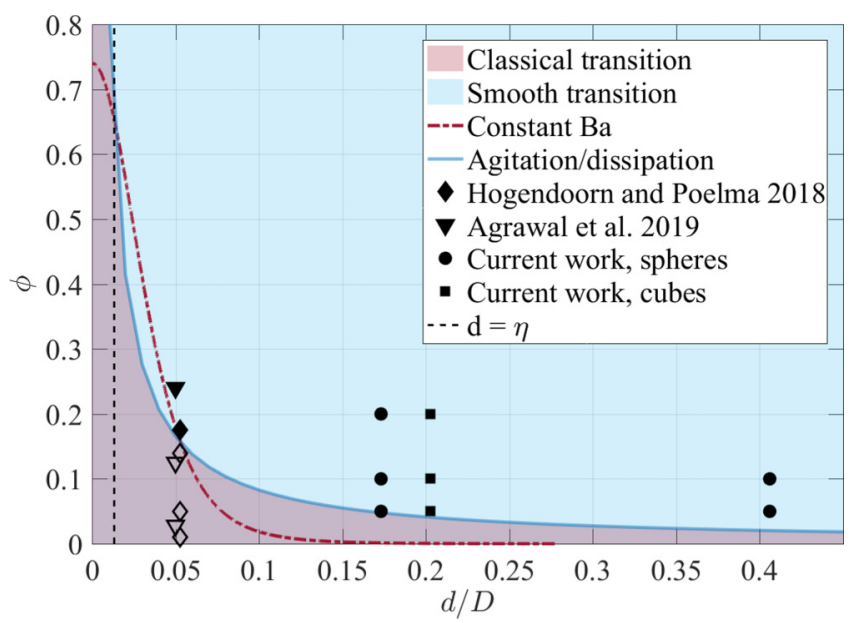

FIG. 3. State space showing classical and smooth transitions where experiments from Hogendoorn and Poelma [13], Agrawal et al. [14], and our own work are plotted. Filled markers indicate smooth transition and empty markers indicate classical transition. The red curve shows constant Bagnold number. The blue curve indicates that the limit between the two regions is derived from a scaling analysis based on particle-agitation to fluid-dissipation ratio. The black dashed line represents the size of the smallest Kolmogorov length scales at a Reynolds number of 1000 . 
be derived assuming laminar flow. This relation shows that at $\operatorname{Re}=1000$, which is the minimum $\operatorname{Re}$ for which turbulence can be observed [2], particles smaller than $d / D \approx 0.013$ will be smaller than the dissipative length scale $\eta$. This particle size is indicated with a dashed vertical line in Fig. 3 and is close to the particle size for which agitation can be expected to be too small to initiate a smooth transition. This is in congruence with the observation of Matas et al. [11], where particles with size $d / D<0.015$ did not alter the classical transition scenario but instead caused an increase in the effective viscosity, thereby delaying the classical transition behavior to higher Re with increasing $\phi$.

Classical transition is a backward bifurcation controlled by the velocities of the upstream and downstream edges of turbulent puffs [8,23]: when the downstream velocity increases above the upstream velocity, the size of the turbulent region is increasing, eventually leading to fully turbulent flow. Our agitation discussion originates from an assumption that the bifurcation is still a backward one, and that the main effect of the particles is to generate disturbances that bring the system to the upper branch of the backward bifurcation whenever this branch exists. However, the particles could also change the nature of the underlying bifurcation, for example, due to the fact that particles can be expected to move both faster (if they are at the centerline) and slower (if they are close to the wall) than turbulent puffs. Particles might therefore move out of turbulent puffs in both directions. If the particles induce fluctuations while doing so, they could contribute to early transition not only by the fluctuations as such, but also by providing a mechanism by which turbulent puffs can be elongated. Understanding particle-puff interactions could be a rewarding aim of future numerical and experimental investigations and one aspect to be studied is which, if any, particle effects that are controlled by our agitation-dissipation relation.

Concluding, experiments have shown that suspensions with large particles in pipe flow exhibit a nonclassical (smooth) transition behavior already at the low concentrations of 5\%. During the smooth transition, the friction factor as a function of $\mathrm{Re}_{e}$ approaches the turbulent friction factor for single-phase flow from above in a monotonic manner via two distinct curves. Large velocity fluctuations are observed at subcritical Re. The observations could be the result of either agitation from particles passing each other in shear or changes in the fundamental dynamics. Attempts to quantify the former and identify mechanisms behind the latter are presented. Further understanding will come from detailed experiments and simulations of suspensions with large particles and low concentrations.

Financial support from The Swedish Energy Agency and Tetra Pak Processing Systems AB is gratefully acknowledged. This work has benefited from discussions with Dr. Sagar Zade and Professor Veronique Roig. We are grateful to one of the reviewers who suggested Fig. 1(b) and helped us improve the discussion substantially.

[1] O. Reynolds, An experimental investigation of the circumstances which determine whether the motion of water shall be direct or sinuous, and of the law of resistance in parallel channels, Philos. Trans. R. Soc. 174, 935 (1883).

[2] T. Mullin, Experimental studies of transition to turbulence in a pipe, Annu. Rev. Fluid Mech. 43, 1 (2011).

[3] D. J. Tritton, Physical Fluid Dynamics (Springer Science \& Business Media, 2012).

[4] I. J. Wygnanski and F. H. Champagne, On transition in a pipe. Part 1. The origin of puffs and slugs and the flow in a turbulent slug, J. Fluid Mech. 59, 281 (1973).

[5] I. Wygnanski, M. Sokolov, and D. Friedman, On transition in a pipe. Part 2. The equilibrium puff, J. Fluid Mech. 69, 283 (1975).

[6] B. Eckhardt, T. M. Schneider, B. Hof, and J. Westerweel, Turbulence transition in pipe flow, Annu. Rev. Fluid Mech. 39, 447 (2007).

[7] M. Avila and B. Hof, Nature of laminar-turbulence intermittency in shear flows, Phys. Rev. E 87, 063012 (2013). 
[8] D. Barkley, B. Song, V. Mukund, G. Lemoult, M. Avila, and B. Hof, The rise of fully turbulent flow, Nature (London) 526, 550 (2015).

[9] R. T. Cerbus, C. C. Liu, G. Gioia, and P. Chakraborty, Laws of Resistance in Transitional Pipe Flows, Phys. Rev. Lett. 120, 054502 (2018).

[10] H. Blasius, Das Ähnlichkeitsgesetz bei Reibungsvorgängen in Flüssigkeiten, Mitteilungen über Forschungsarbeiten auf dem Gebiete des Ingenieurwesens (Springer, 1913), pp. 1-41.

[11] J.-P. Matas, J. F. Morris, and É. Guazzelli, Transition to Turbulence in Particulate Pipe Flow, Phys. Rev. Lett. 90, 014501 (2003).

[12] Z. Yu, T. Wu, X. Shao, and J. Lin, Numerical studies of the effects of large neutrally buoyant particles on the flow instability and transition to turbulence in pipe flow, Phys. Fluids 25, 043305 (2013).

[13] W. Hogendoorn and C. Poelma, Particle-Laden Pipe Flows at High Volume Fractions Show Transition Without Puffs, Phys. Rev. Lett. 121, 194501 (2018).

[14] N. Agrawal, G. H. Choueiri, and B. Hof, Transition to Turbulence in Particle Laden Flows, Phys. Rev. Lett. 122, 114502 (2019).

[15] R. A. Gore and C. T. Crowe, Modulation of turbulence by a dispersed phase, J. Fluids Eng. 113, 304 (1991).

[16] S. Balachandar and J. K. Eaton, Turbulent dispersed multiphase flow, Annu. Rev. Fluid Mech. 42, 111 (2010).

[17] I. Lashgari, F. Picano, W.-P. Breugem, and L. Brandt, Laminar, Turbulent, and Inertial Shear-Thickening Regimes in Channel Flow of Neutrally Buoyant Particle Suspensions, Phys. Rev. Lett. 113, 254502 (2014).

[18] R. A. Bagnold, Experiments on a gravity-free dispersion of large solid spheres in a newtonian fluid under shear, Proc. R. Soc. London, Ser. A 225, 49 (1954).

[19] J. J. Stickel and R. L. Powell, Fluid mechanics and rheology of dense suspensions, Annu. Rev. Fluid Mech. 37, 129 (2005).

[20] P. Dyverfeldt, A. Sigfridsson, J. P. E. Kvitting, and T. Ebbers, Quantification of intravoxel velocity standard deviation and turbulence intensity by generalizing phase-contrast MRI, Magn. Reson. Med. 56, 850 (2006).

[21] C. J. Elkins and M. T. Alley, Magnetic resonance velocimetry: Applications of magnetic resonance imaging in the measurement of fluid motion, Exp. Fluids 43, 823 (2007).

[22] J. MacKenzie, D. Söderberg, A. Swerin, and F. Lundell, Turbulent stress measurements with phasecontrast magnetic resonance through tilted slices, Exp. Fluids 58, 51 (2017).

[23] D. Barkley, Theoretical perspective on the route to turbulence in a pipe, J. Fluid Mech. 803, 1 (2016). 